Meta

Journal des traducteurs

Translators' Journal

\title{
Structures du lexique
}

\section{Josette Rey-Debove}

Volume 18, numéro 1-2, mars 1973

Actes du deuxième colloque international de linguistique et de traduction. Montréal, 4-7 octobre 1972

URI : https://id.erudit.org/iderudit/002494ar

DOI : https://doi.org/10.7202/002494ar

Aller au sommaire du numéro

Éditeur(s)

Les Presses de l'Université de Montréal

ISSN

0026-0452 (imprimé)

1492-1421 (numérique)

Découvrir la revue

Citer cet article

Rey-Debove, J. (1973). Structures du lexique. Meta, 18(1-2), 53-60.

https://doi.org/10.7202/002494ar

Ce document est protégé par la loi sur le droit d'auteur. L'utilisation des services d'Érudit (y compris la reproduction) est assujettie à sa politique d'utilisation que vous pouvez consulter en ligne.

https://apropos.erudit.org/fr/usagers/politique-dutilisation/
Cet article est diffusé et préservé par Érudit.

Érudit est un consortium interuniversitaire sans but lucratif composé de l’Université de Montréal, l'Université Laval et l'Université du Québec à Montréal. Il a pour mission la promotion et la valorisation de la recherche. https://www.erudit.org/fr/ 


\section{Structures du lexique}

Le lexique, on l'a dit à juste titre, est la somme des irrégularités d'une langue. Chacun sait pourtant que des régularités s'y manifestent, et que c'est pour en parler que nous sommes ici. Qu'on y repère des régularités ou des irrégularités, il est certain en tout cas que le lexique n'est pas homogène et qu'il offre, globalement, des zones inégalement structurées.

Je voudrais caractériser la zone de plus faible structuration afin de voir ce qui s'y passe et faire porter les efforts de la recherche de ce côté-là. Plutôt que d'envisager ce qu'on peut ramener à des régularités dans le lexique, j'aborderai d'emblée ce qui, à l'évidence, est impossible à décrire, tout au moins avec les moyens linguistiques dont nous disposons actuellement. Ceci afin de saisir les difficultés là où elles se manifestent le mieux, en gardant à l'esprit que ces causes de déstructuration se répercutent dans la solidarité totale du lexique.

Il y a trois définitions courantes du lexique orientées soit sur le choix entre le morphème et le mot (la plus haute des unités codées qui n'est pas une phrase), soit sur le rejet des unités de classe fermée dites grammaticales qu'on oppose aux unités de classe ouverte dites lexicales : 1) ensemble des morphèmes (libres et liés) ; 2) ensemble des mots ; 3 ) ensemble des unités de classe ouverte (morphèmes et mots, ou mots seulement).

La première oppose clairement les règles (la grammaire) aux unités (le lexique) mais ne convient pas au lexicologue, car les assemblages de morphèmes selon le système produisent des mots inconnus de la norme. La deuxième renvoie tous les morphèmes liés à la grammaire, ce qui est peu satisfaisant. La troisième est peu claire car les ensembles fermés existent partout, y compris parmi les mots lexicaux (par exemple : noms des doigts, des grades militaires, etc.), et elle exclut trop d'unités. Je choisirai une quatrième définition cumulant les deux premières parce que c'est la plus large, qu'elle oppose les règles aux unités et qu'elle rend compte des deux niveaux d'intégrants, le mot et le morphème, mis en valeur par Benveniste : l'ensemble des morphèmes liés et des mots.

D'une façon classique j'envisagerai pour ce lexique les types de structures suivantes : 1) structures syntaxiques; 2) structures morpho-sémantiques ; 3) structures purement sémantiques; 4) structures phonologiques. Mais on peut admettre que ces structures sont dominées par les structures sémiotiques générales qui régissent le système signifiant de la langue dans ses rapports avec les autres 
systèmes : $1^{\circ}$ autres systèmes linguistiques ou paralinguistiques, $2^{\circ}$ système socioculturel du monde (les «choses», dans l'opposition «les mots et les choses»). Ces structures fondamentales reposent sur : l'arbitraire du signe; la double articulation ; la finitude de l'ensemble lexical.

Je voudrais montrer ici que ces bases de la linguistique sont mises en défaut à cause du NOM, dans la mesure où c'est un substantif (noun) et une dénomination (name). Le nom est la partie du discours qui réalise le mieux l'idée que nous nous faisons du lexique (témoin : les exemples des lexicologues et sémanticiens) et c'est aussi la moins linguistique, celle qui gêne le plus la description. Le nom se trouve très étroitement lié aux autres systèmes :

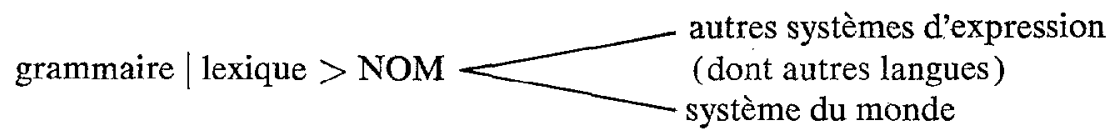

J'envisagerai le phénomène nominal dans sa totalité :

ensemble des noms

(code) noms communs ex. : table «meuble»

(hors code lexical) noms propres ex. : Hjelmslev «le linguiste $\gg$

(hors code lexical) noms autonymes ex. : table «le mot table », Hjelmslev « le mot Hjelmslev »

Cet ensemble lexical est quantitativement sans commune mesure avec l'ensemble formé par les autres parties du discours. Cotmme conséquence préliminaire, on doit admettre que cet ensemble contiendra les mots les plus rares, donc les mots les plus atypiques d'une langue donnée.

On commencera par décrire les propriétés des noms propres et des noms autonymes, qui sont hors code, donc les plus aberrants dans la langue.

\section{LE NOM PROPRE}

Cet ensemble est hors code lexical ; il se situe hors de la compétence lexicale de l'usager. Il est formé de tous les noms de personnes, de lieux, de productions (notamment titres d'cuvres), etc. de tous les pays et de tous les temps. On peut le caractériser comme interlingual et achronique (avec quelques aménagements grapho-phoniques). C'est un ensemble indéterminé. Cependant on constate sa présence dans n'importe quelle phrase bien formée (donc sémantique) d'une langue donnée. Pour cette raison il a forcément un signifié : Hjelmslev signifie «celui qui s'appelle Hjelmslev». Or, si un nom (mot autonyme) fait partie d'un signifié, il n'existe ni synonyme ni traduction, et d'une certaine façon, l'arbitraire du signe est en défaut (remarque : autrefois on traduisait certains noms propres morphologiquement motivés, Den Haag $\rightarrow$ La Haye). Par ailleurs, le nom propre perturbe le lexique commun dans la mesure où il se lexicalise en nom commun (boire un Martini en fumant une Pall Mall) ou produit des dérivés (Nietzsche $\rightarrow$ nietzschéen).

Effets syntaxiques : règles spéciales d'emploi du nom propre. 
Effets morphologiques : règles spéciales (en français pas de marque du pluriel). Opacité morpho-sémantique des dérivés dont la racine est hors code (nietzschéen).

Effets sémantiques : indétermination et ouverture de l'ensemble. Signifié partiellement opaque ( «celui qui s'appelle Hjelmslev» ne nous fait pas connaitre le linguiste). Indétermination et ouverture de l'ensemble des noms de marques, des dérivés de noms propres.

Effets phonologiques : irruption du phonétisme étranger par les noms propres, leurs produits lexicalisés ou dérivés.

\title{
NOMS COMMUNS
}

Exemples : les mots table, peut-être, white tels qu'ils figurent dans les énoncés «table est monosyllabique », «ses peut-être m'exaspèrent», «white est un mot angiais $»$.

Comme les noms propres, ils sont hors code lexical, mais de différente façon. Ils sont interlinguaux aussi, mais un sous-ensemble est intralingual et homonyme du lexique ordinaire (table «meuble» et table «le mot table»). D'autre part, des éléments de systèmes non linguistiques peuvent apparaître : « la formule $\pi \mathrm{R}^{2}$ est celle de la surface du cercle ». «Il fait [geste] ». Les noms autonymes, enfin, sont issus de toutes les parties du discours, de toutes les unités linguistiques et même des «figures » bloomfieldiennes dont ils sont homonymes :

\author{
ou indique la disjonction \\ ses peut-être m'agacent \\ il dit I'm coming \\ les rimes en eur \\ la phrase se termine par et sans aucun regret
}

La compétence pour les noms autonymes serait celle de toutes les langues et de tous les systèmes d'expression susceptibles de s'insérer dans le discours. Cependant, comme dans le cas des noms propres, les noms autonymes apparaissent normalement dans toute phrase bien formée. Nous linguistes, produisons quotidiennement ce type de phrase irréprochable. Les noms autonymes, dans leur sous-ensemble intralingual, diffèrent des noms propres dans la mesure où, bien que hors code lexical, ils relèvent totalement du système de la langue. Car toute langue a un métalangage autonyme (et un autre, non autonyme, qui ne nous intéresse pas ici) qui lui permet de parler d'elle-même. Si la compétence linguistique pour une langue $L$ est idéale, la compétence métalinguistique y est obligatoirement impliquée : qui connaît table «meuble » connaît forcément table « mot qui signifie meuble ». Pour les mots autonymes étrangers, comme white en français, le signifié est partiellement opaque ainsi que dans le cas du nom propre; white signifie «le mot dont le nom est white», « le mot white qui signifie ? ». Que le nom autonyme appartienne à la langue qui l'emploie ou non, il se trouve que son nom est présent dans son signifié et que comme le nom propre, il n'a ni synonyme ni traduction, mettant en échec l'arbitraire du signe : 
white est un mot anglais $\rightarrow$ white és un vocablo inglés

Il met aussi en cause le principe de la double articulation dans la mesure où les unités de seconde articulation passent en homonymes dans la première (les rimes en eur, $a$ est une lettre). Heureusement pour le système de la langue, les noms autonymes se lexicalisent rarement et ne produisent guère de dérivés (en français $n^{\text {ieme }}, j$ 'menfoutisme, le nom de certaines lettres). Par contre l'autonymie est le lieu de passage de l'emprunt lexical, tout emprunt commençant par être un nom autonyme, puis un mot du lexique :

Mot autonyme : les Anglais chassent le coq de bruyère qu'ils appellent grouse.

Mot à connotation autonymique : les Anglais chassent la grouse, comme ils appellent le coq de bruyère.

Mot ordinaire : les Anglais chassent la grouse.

Effets syntaxiques : règles spéciales d'emploi du nom autonyme (pas d'article en français).

Effets morphologiques : règles spéciales voisines de celles du nom propre. Pas de marque du pluriel en français (vos Monsieur sont trop cérémonieux).

Effets sémantiques : indétermination et ouverture de l'ensemble autonyme. Signifié partiellement opaque pour les mots étrangers.

Effets phonologiques : irruption des systèmes phonologiques étrangers avec passage dans le lexique en cas d'emprunt.

\section{NOMS COMMUNS}

Ils font partie du code lexical, mais sont en quantité si nombreuse qu'ils recèlent par définition les mots les plus rares donc les plus atypiques. II semble que dans toutes les langues le nom (noun) soit la partie du discours qui forme de loin l'ensemble le plus vaste.

Le nom commun offre des caractères spéciaux auxquels nous devons accorder plus d'attention : $a$ )il est la première acquisition de l'enfant dans l'apprentissage de la langue (Tabouret-Keller) ; b) il forme le stock des object-words (Russel) du langage primaire (langage qui parle des objets du monde), par opposition aux autres niveaux du langage ; c) il exprime une substance (Aristote, Chomsky) et constitue la partie du discours qui est la plus indépendante du contexte (syntaxique et sémantique) ; le code sature son signifié, ou si l'on veut, la valeur de l'occurrence est très proche de la valeur de l'unité de langue.

La majorité des mots d'emprunt dans la langue sont des noms communs (entre 65 et $80 \%$ selon les couples de langues).

Effets syntaxiques : c'est la partie du discours la moins grammaticalisée ; sa distribution est la moins nette (difficultés des distributionnalistes et des lexicographes), ne dépendant ni d'un sujet ni d'un objet. En français le verbe, l'adjectif et l'adverbe se nominalisent (sans changement de forme); le nom, l'adjectif et l'adverbe ne peuvent se verbaliser. Toute séquence autonyme lexicalisée est un nom. 
Effets morphologiques : les morphèmes étrangers s'introduisent par le nom qui représente la plus grande masse des éléments empruntés (par exemple, en français les morphèmes anglais -ing et -man dans parking et tennisman). Beaucoup de noms étrangers ne suivent plus les règles du système (par exemple, en français absence de $s$ du pluriel et alternances étrangères au système concerto/concerti, cameraman/cameramen). Les sigles sont typiquement nominaux (H.L.M., C.G.T., etc.) et morphologiquement aberrants (les lettres initiales étant métalinguistiques). De plus ils produisent des dérivés (cégétiste).

Effets sémantiques : indétermination et ouverture de l'ensemble des noms communs liées à leur aspect substantiel et à leur relation étroite avec les objets du monde qu'ils dénomment. La grande majorité des noms forme les taxa, les terminologies (la plupart des termes techniques et scientifiques). Ils représentent le domaine de l'encyclopédie; témoin : les nomenclatures des encyclopédies opposées aux dictionnaires de langue. Les noms représentent la partie du discours la plus mobile et la plus labile, étant liés aux stades successifs de dénomination qui reflète les stades de la connaissance (objets nouveaux ou restructuration des ensembles anciens objets). C'est là que la néologie et l'emprunt se manifestent surtout. Le type d'unité qui échappe à l'arbitraire du signe, l'onomatopée, est essentiellement représentée par le nom. L'onomatopée étant une icône, on connâit son signifié si le signifiant est présent, quelle que soit la compétence réelle du décodeur. Elle produit des dérivés (glouglou $\rightarrow$ gouglouter). Elle est mal codée (on en invente selon les besoins). Son sémantisme s'apparente à celui du mot autonyme étranger, mais le signifié de l'onomatopée est complet : «Le chat fait miaou. » "César dit Alea jacta est. » L'onomatopée fait pénétrer dans les langues tous les bruits du monde. Les noms communs issus de noms propres ont un signifié partiellement opaque (kantisme). L'analyse du sens trouve sa contradiction dans le nom ; partie du discours la moins grammaticalisée, la plus indépendante du contexte, le nom se prête aisément à l'analyse sémantique (synonyme péri-phrastique) ; cependant, élément d'un ensemble indéterminé et ouvert, il se prête mal à la recherche des traits sémantiques différentiels pertinents, sauf dans quelques microsystèmes fermés. D'où le statut de la sémantique lexicale.

Effets phonologiques : les structures phonologiques sont surtout ébranlées dans le nom par le triple apport des onomatopées (bruits), des sigles et des mots étrangers (dérivés de noms propres étrangers, noms propres étrangers lexicalisés, noms étrangers empruntés). Ainsi en français le passage des phénomènes [n] de -ing, [h] de home, $[\mathrm{X}]$ de jota, khamsin. Aussi la perturbation des relations graphophoniques oo [u] dans boom, er [œR] dans mixer, $u$ [œ] dans $c l u b$, etc. (rejet du mot graphique). D'autre part les mots les plus rares (de quelque provenance) sont des noms, et les mots rares sont surtout des mots lus. D'où la prédominance du svstème graphique (rédemption $\rightarrow$ [Redũpsjõ]) et une perturbation inverse de la précédente dans les relations grapho-phoniques (rejet du mot phonique).

J'espère avoir évoqué ici, bien que cursivement, comment les irrégularités du lexique semblent se concentrer dans le nom comme partie du discours (noun) et comme dénomination (name). Si l'on considère, comme le faisait ce matin E. Coseriu, que les mots «lexicaux» sont tous des noms (names), je pense que 
parmi ces dénominations c'est la dénomination substantive (noun) qui ouvre dans le lexique la brèche par laquelle pénètrent et agissent les éléments extérieurs à la langue. C'est ce nom (noun/name) qui rend particulièrement difficile la description des structures du lexique.

Josette Rey-Debove

\section{QUESTIONS}

M. Guilbert : Je veux poser deux questions. Une première, sur le signifié du nom propre. Vous avez dit que le signifié du nom propre se résumait à la personne désignée. Ne pensezvous pas que le nom propre peut avoir un autre signifié ? $\mathrm{Si}$ je prends, par exemple, un bottin de Montréal et que je parcours la liste des noms, ces noms propres, ces personnes, je ne les connais pas, mais je suis capable de distinguer un nom anglais d'un nom français. Par conséquent, par le signifiant même, le signifiant du nom propre a une certaine signification pour celui qui a un degré tout à fait élémentaire de connaissance des langues et même pour celui qui n'a aucune connaissance des langues, il a au moins la signification, par exemple, selon l'état de la langue.

Réponse : Je peux répondre que ce n'est pas typique du nom propre, c'est typique du mot inconnu. Vous avez une information quand vous voyez un nom propre. Est-ce français? Est-ce canadien? Est-ce anglais ? Est-ce mexicain ? C'est vrai aussi quand vous rencontrez un nom inconnu, un mot du lexique.

M. Guilbert : La deuxième question porte sur le nom commun. Vous avez dit que le nom commun avait comme caractéristique sa faculté de capitaliser les différentes catégories grammaticales, et que l'inverse n'était pas vrai. La catégorie du nom a cette faculté effectivement de recevoir dans son sein à peu près toutes les autres catégories, mais que l'inverse n'est pas vrai. C'est une affirmation que je conteste en partie. Si on résume l'essentiel des catégories lexicales au nom, à l'adjectif et au verbe, on peut remarquer que pour chacune d'entre elles, il y a une faculté de passage à l'autre catégorie. Un nom peut devenir verbe, un nom peut devenir adjectif, un adjectif peut devenir verbe, un adjectif peut devenir nom, un verbe peut devenir nom et un verbe peut devenir adjectif. Par conséquent, il me semble qu'il y a au contraire une permutation générale possible.
Réponse : Excusez-moi, je me suis fait mal comprendre, je n'ai pas parlé de dérivé, jaai voulu parler de passage sans aucun avis morphologique, car je ne peux pas passer d'un nom à un verbe directement. Cela dépend évidemment de chaque langue, mais je crois que le phénomène de nominalisation doit être général dans les langues, alors que dans l'autre sens, cela dépend de chaque système.

M. Coseriu : J'ai deux petites observations à faire. En ce qui concerne les noms propres, je crois qu'il faudrait plutôt parler d'une couche dans le langage constituée par des désignations d'identifications historiques, où on a les noms propres substantifs, les adjectifs propres, les adverbes propres, le verbe propre, par exemple italianiser, russifier, etc., où on a des substantifs abstraits propres, des pronoms propres, etc. Il s'agit en réalité non pas du nom propre, mais d'une couche du langage tout entier qui est cette couche d'identification historique. Il est vrai qu'en général, ces adjectifs, ces adverbes propres, ces verbes propres procèdent de substantifs propres, c'est-à-dire qu'on a, par exemple italia et ensuite italiano, italianamente, etc., mais ceci n'est pas nécessaire. Il paraît que dans certains cas l'adjectif propre se trouve à l'origine et qu'on ait eu ensuite des substantifs propres.

Deuxième petite remarque : vous avez très bien observé les caractères spécifiques du métalangage et, en particulier, le fait qu'il y a des règles grammaticales propres à une langue donnée pour l'emploi métalinguistique. Mais vous n'avez considéré que le métalangage, que moi-même j'appelle métalangage du discours, c'est-à-dire ce métalangage qui permet à n'importe quel moment de parler, par exemple d'une partie d'un mot ou de la première lettre de mots écrits, etc. Or il y a aussi un métalangage de langue et qu'il faut considérer comme métalangage, mais qui ne suit pas ces règles parce que, puisque le langage appartient aussi à la réalité, alors le langage même est 
aussi classé par les langues mêmes et par des terminologies et on a toute une série de mots qui sont métalinguistiques, qui sont nos expressions, c'est-à-dire le mot nom, par exemple, ou parole, boniment, discours, etc. Ce sont toujours des noms d'expression. Alors à l'époque de la Renaissance déjà, on distinguait dans ces sens de façon très claire les rerum nomina et les verborum nomina. Le métalangage dont parle saint Augustin, c'est ce métalangage de langue. Il oppose, par exemple, plumen à verbum.

Réponse : Je n'ai abordé absolument dans le phénomène métalinguistique que l'aspect autonymique, c'est-à-dire restrictif. Dans le lexique par exemple, on peut considérer qu'il y a deux séries métalinguistiques. Une série qui est métalinguistique par son signifié uniquement, mais qui ne présente aucun caractère syntaxique ou morphologique spécial qui serait la série : nom, verbe, préposition, poème, conjugaison; tous les noms et tous les mots mêmes qui parlent de la langue, mais qui ne sont pas autonymes, c'est-à-dire qui ne se signifient pas eux-mêmes. Préposition signifie, au contraire, la classe de : à, de, par, pour, sans, etc. Alors que table est un nom féminin, table se signifie lui-même. C'est donc, pour moi, ce qu'il y a d'important dans le métalangage, c'est l'aspect autonymique, l'aspect restrictif. Mais il est certain que l'autre aspect est très important aussi. Il a aussi un rapport avec la notion de propre, nom propre, adjectif propre, verbe propre. Il y a une chose qui m'ennuie, c'est que l'emploi de noms propres, pour moi, est lié à un référent singulier opposé à une classe, le singulier opposé au général. Je ne suis pas philosophe du langage, je ne sais pas ce qu'il faut penser du singulier et du général pour l'adjectif, pour le verbe, pour l'adverbe. Est-ce que selon vous, cette distinction singulier, général, continue à se manifester dans l'adjectif propre? Oui.

M. Querido : Dans l'ensemble, je suis d'accord avec les remarques que vous avez faites sur le nom, mais je pense que quelques-unes n'auraient de sens que si l'on prend le nom exclusivement dans une des principales fonctions syntaxiques qu'il peut jouer. Mais vous savez très bien que le nom commun fonctionne aussí comme prédicat et à ce momentlà, par exemple, des remarques, comme le nom dénote la substance opposée au verbe, n'auraient pas de sens parce que le nom serait en opposition complémentaire avec le verbe et la remarque que vous avez faite sur l'indépendance du contexte du nom tomberait aussi.

Réponse : L'objet dont on parle, c'est toujours forcément un nom et la langue peut parler de n'importe quoi. C'est ça mon point de vue. N'importe quoi, c'est forcément un nom.

M. Querido : Vous vous êtes concentrée sur la fonction principale du nom. Mais il reste que vous avez passé sous silence tous les autres emplois.

Réponse : Oui, ça je le reconnais. Seulement, c'était cela qui m'intéressait surtout.

M. Querido : D'ailleurs, pour le nom commun, on a tendance, par exemple, à faire porter le référent sur une variable référentielle. On pourrait remplacer «appelle » par « est » et dire que le signifié du nom commun voisin, par exemple, c'est "celui qui est proche de ", ou «qui est voisin de ". Tenez, par exemple, un nom nettement transitif comme "père de ". Vous ne direz pas que dans "Jean est le père de Paul ", père dénote une substance. Il dénote une relation.

Réponse : Oui, c'est juste, mais j’ai dit simplement que c'était parmi les noms qu'il y avait les mots les plus rares. Je dis que c'est parmi les mots qu'il y a les dénominations et que c'est un phénomène important. Je reconnais évidemment qu'il y a d'autres types.

M. Coseriu : Deux petites observations : tout d'abord, le fait que le substantif puisse se trouver dans le prédicat ne signifie pas qu'il ne désigne pas une substance, parce que la substance peut parfaitement se trouver dans le prédicat et Aristote qui est le créateur de la notion de substance a précisé qu'il y a des substances qui peuvent se trouver uniquement dans le sujet, d'autres peuvent se trouver uniquement dans le prédicat et d'autres encore peuvent se trouver aussi bien dans le sujet que dans le prédicat. Deuxièmement, je voudrais maintenir l'idée de substance de $M^{\text {me }}$ Rey, parce que «substance 》 ne signifie pas, dans la définition du nom, une chose matérielle, une substance matérielle, voir à ce sujet une définition bien connue donnée par Spinoza.

Le « père » dans ce sens est une substance et le "blanc", si vous dites le "blanc " c'est une substance et " le fait d'arriver ", c'est une substance, etc.

M. Querido : Je me refuse à vous suivre sur ce chemin, nous laissons la linguistique pour entrer dans la métaphysique. 
M. A. Bachrach : Vous avez dit que vous n'acceptez pas les noms propres dans le lexique et que les noms propres n'avaient pas de traduction. Mais qu'est-ce que vous faites, par exemple, du mot, du nom propre «Hoover» et qui a tout de même en français pour traduction " aspirateur .

Réponse : Quand \& Hoover » désigne un aspirateur, ce n'est plus un nom propre. C'est lexicalisé, c'est passé dans le code.

M. Bachrach : L'allusion Hoover n'est pas nécessairement un aspirateur. On peut penser à un président des États-Unis ou autre chose.

Réponse : Oui, mais là vous faites allusion à un problème théorique, celui de la compétence. Pour progresser dans la recherche, je pense qu'il faut toujours envisager une compétence parfaite, idéale. Les gens connaissent toute la grammaire, tous les mots de leur langue. Si on commence à dire certains ne connaissent pas tel mot, on tombe dans la psycho-linguistique. 